\title{
Integrative biology and systems biology
}

\author{
Molecular Systems Biology 29 March 2005; doi:10.1038/msb4100008
}

Demarcations of scientific fields have always followed either a conceptual breakthrough or have been a result of profoundly enabling technologies. Quantum theory and quantum mechanics revolutionized physics and engineering, but highenergy physics is a discipline fundamentally enabled by the linear accelerator. Similarly, the discovery of the structure of DNA gave birth to molecular biology; structural biology is completely dependent on the technologies of chemical analysis albeit on complex molecules, and genomics was born out of high-throughput DNA sequencing (arguably, the most important being sequencing by capillary electrophoresis). No naming-committee was ever established to set the definitions for these disciplines; instead the fields arose from a consensus of the scientific community often driven by individual champions, and like cultural movements, only recognized in retrospect.

The launch of the journal Molecular Systems Biology is a strong indication that another field has emerged: that of systems biology. Other evidence of its emergence is extant: articles with 'systems biology' in its abstract are increasing, and new departments of systems biology are being established in academic centers. As editors for this new enterprise, we were asked to give our personal views as to the meaning of systems biology to help define the field and to frame the contents of the inaugural issues.

To me, systems biology seeks to explain biological phenomenon not on a gene-by-gene basis, but through the interaction of all the cellular and biochemical components in a cell or an organism. Since, biologists have always sought to understand the mechanisms sustaining living systems, solutions arising from systems biology have always been the goal in biology. Previously, however, we did not have the knowledge or the tools.
The fundamental characteristics of systems biology are embodied in data and knowledge integration, the comprehensiveness of the data acquisition, and the ability to digitalize biological output. Integrative biology and digital biology may be considered synonyms. The enabling technologies for systems approaches have been the human genome sequence that serves as the integrating principle and the means to digitalize biological information, and advanced computational capabilities, which allow for the analysis of such comprehensive and complex data. I do believe, however, that the ultimate goal will be the ability to predict de novo biologic processes given the list of components involved; that is, predictive biology.

How we accomplish this state of predictive biology is precisely what we are pursuing in this journal of Molecular Systems Biology: novel approaches to systems integration; computational simulations backed by experimental validation; precise determination of the cellular components and their quantitative assessment; and deterministic models of complex biological processes. Along the way, we will need to pause to develop new terminologies, and to educate ourselves in disciplines not within the domain of classical biology.

As a scientist, the systems approach is very empowering and moves us closer to a formal theoretical framework for biomedical investigations. As a physician, I am very excited that a systems approach will help explain the complexities of human disease and the vagaries of our therapies. It will surely move decision-making in clinical medicine from its empiric approach to one that is truly mechanistically driven.

Edison T Liu Genome Institute of Singapore 